\title{
EVALUATION OF THE RADIOMETRIC ACCURACY OF IMAGES OBTAINED BY A SEQUOIA MULTISPECTRAL CAMERA
}

\author{
Alison A. D. Teixeira ${ }^{1}$, Cláudio W. Mendes Júnior ${ }^{1 *}$, Christian Bredemeier ${ }^{1}$, \\ Marcelo Negreiros ${ }^{1}$, Robson dos S. Aquino ${ }^{1}$
}

$1^{1 *}$ Corresponding author. Federal University of Rio Grande do Sul/ Porto Alegre - RS, Brazil.
E-mail: claudio.mendes@ @ufrgs.br | ORCID ID: https://orcid.org/0000-0003-1745-348X

\section{KEYWORDS}

NDVI, NDRE,

Sequoia camera, radiometric correction.

\begin{abstract}
Geometric and radiometric corrections of images obtained by remotely piloted aircraft systems (RPAS) are required for the measurement of the spectral reflectance and calculation of vegetation indices. This study aimed to determine which radiometric calibration procedures allow obtaining suborbital images with more accurate reflectance data and spectral indices based on data collected by a Sequoia multispectral camera coupled to a RPAS on black oat cultivations at the flowering stage located in Eldorado do Sul, RS, Brazil. The orthophoto mosaics were processed without and with radiometric corrections by reflectance calibration ground target and/or solar irradiance sensor of the camera, and the radiometric accuracy of these images was evaluated using spectral reflectance ground data collected by the Crop Circle canopy sensor. The results indicated that the use of a reflectance calibration ground target is recommended to increase the radiometric accuracy of photographs even if the Sequoia solar irradiance sensor is employed. The use of the light gray target in the radiometric calibration resulted in orthophotos with the mean square error of reflectance values lower than 0.01 for all the analyzed bands and spectral indices.
\end{abstract}

\section{INTRODUCTION}

Remotely piloted aircraft systems (RPAS) have been used since the beginning of the 21 st century as multispectral sensor platforms, which can be used to acquire spectral reflectance data of crops (Castro \& Inamasu, 2014). These radiometric measurements can be used to calculate vegetation indices (VI) that can be applied to evaluate the biophysical characteristics of plants, which is an important ally in precision agriculture (PA) (Pereira et al., 2016). The Normalized Difference Vegetation Index (NDVI), calculated by the ratio of the difference between the reflectance in the near-infrared (NIR) band and the red band by the sum of the reflectance of these spectral bands, is one of the most used (Rouse et al., 1974). This VI is influenced by the biophysical characteristics of the plant and can be used to analyze plant stresses (Turra, 2016). Another VI that has been gaining prominence is the Normalized Difference Red Edge Index (NDRE), calculated by the ratio of the difference between the reflectance in the NIR band and the red edge (RE) one by the sum of the reflectance of these spectral bands (Barnes et al., 2000). The RE position is influenced by pigments present in the leaves of the vegetation (e.g., chlorophyll), which alter the vegetation spectral curve in this region (Doumit \& Kiselevm, 2017).

Errors resulting from variations in cloud cover, lighting geometry, and viewing geometry of the sensor during data acquisition need to be corrected for the radiometric analysis of plants using sensors on suborbital platforms, such as a RPAS. These corrections are essential for applying these reflectance data and spectral indices in crop monitoring. In addition, the absolute and relative spectral reflectance errors in the NDVI and NDRE of plants of a given crop may be significant with no radiometric corrections in the images acquired by cameras coupled to a RPAS. Thus, studies should be carried out to evaluate quantitatively the results obtained using different radiometric calibration procedures (irradiance sensor and calibration targets) of sensors coupled to a RPAS.

\footnotetext{
${ }^{1}$ Federal University of Rio Grande do Sul/ Porto Alegre - RS, Brazil.
} 
The largest number of studies in which remote sensor images have been used for NDVI and NDRE calculations covers soybean, wheat, and corn crops. Doumit \& Kiselevm (2017) demonstrated the use and potential of VIs for classifying RapidEye images for soybean, wheat, barley, and white oat crops. Damian et al. (2020) used NDVI time series to delimit management zones in three areas of the State of Rio Grande do Sul, along with soybean, wheat, corn, and white oat yield maps. However, there are no studies on the spectral behavior and VIs for black oat.

This study aimed to determine which radiometric calibration procedures allow obtaining suborbital images with more accurate reflectance data and spectral indices based on data collected by a multispectral camera coupled to a RPAS on black oat cultivations at the flowering stage.

\section{MATERIAL AND METHODS}

Black oat cultivations were used to measure the reflectance of plants in the red, red edge and near-infrared spectral bands, and for NDVI and NDRE indices. These radiometric measurements were obtained using a multispectral camera coupled to a RPAS and, subsequently, the radiometric accuracy of suborbital images without and with radiometric calibration was evaluated using data from these bands and spectral indices measured by a canopy sensor.

This study was conducted at the Experimental Agronomic Station of the Federal University of Rio Grande do Sul (EEA/UFRGS) (between latitudes $-30.073800^{\circ}$ and $-30.124030^{\circ}$ and longitudes $-51.651700^{\circ}$ and $-51.703500^{\circ}$ ), located in Eldorado do Sul, RS, Brazil. Data acquisition was carried out on October 4, 2018, from two black oat (Avena strigosa Schreb.) cultivation fields at the beginning of flowering (phenological stage 60) (Zadoks et al., 1974), one of them covering an area of 5 ha and the other 2.5 ha. No precipitation was observed on October 3 and 4, 2018, according to data from an automatic weather station located at the EEA/UFRGS (latitude $-30.096500^{\circ}$ and longitude $-51.673900^{\circ}$; altitude $32 \mathrm{~m}$; Datum World Geodetic System 1984 - WGS84).

The camera used for aerial mapping was the Sequoia model (Parrot Drones SAS) (Figure 1A), which has four spectral bands, two of which at visible wavelengths (Green: 530-570 nm and Red: 640-680 nm), a red edge (RE) band (730-740 nm), and a near-infrared (NIR) band (770-810 nm). The images of these four bands have a 1.2-megapixel resolution and are stored with a radiometric resolution of 16 bits. This multispectral camera has a horizontal field of view (HFOV) of $61.9^{\circ}$, a vertical field of view (VFOV) of $48.5^{\circ}$, and a diagonal field of view (DFOV) of $73.7^{\circ}$ (Deng et al., 2018). In addition, it has a sunshine sensor (SS) that records solar irradiance data for each spectral band when acquiring each image, being used for the radiometric calibration of multispectral photographs taken by the Sequoia camera.

The Sequoia camera was coupled to a DJI Matrice 100 quadcopter (Figure 1B), which is a RPAS capable of providing a stable and reliable flight with resistance to winds up to $10 \mathrm{~m} / \mathrm{s}$. It has an automatic navigation system by Global Navigation Satellite System (GNSS). More information about the DJI Matrice 100 is available in Puri et al. (2017). A MAPIR V2 ground target was used for the radiometric calibration of the images acquired by the Sequoia camera (Figure 1C). It is a reflectance calibration ground target (RC), which has white, light gray, dark gray, and black targets. The reflectance measurements of each of these targets were made by the manufacturer using several Shimadzu integrating sphere spectrophotometers, used to obtain continuous diffuse reflectance (i.e., electromagnetic radiation or EMR scattered from each target) and total data (i.e., including the diffuse and specular reflections from each target). The data of calibrated diffuse reflectance allow the radiometric correction of images obtained from any tilt angle of the camera. Moreover, they can be used for radiometric calibration of images obtained by any camera that operates in the spectral ranges from 350 to 1,094 nm (MAPIR, 2020). 


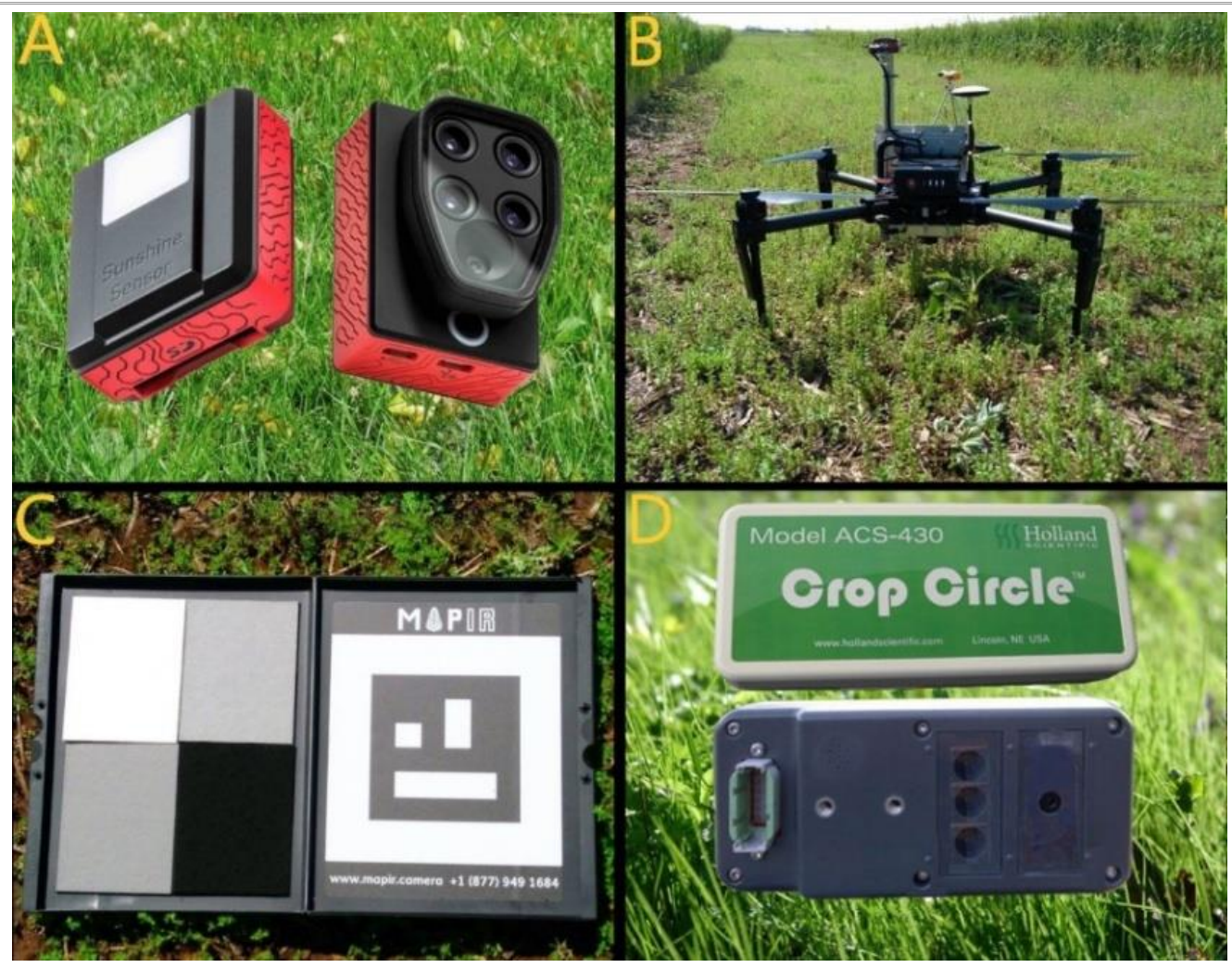

FIGURE 1. Equipment used in this experiment: (A) Sequoia camera; (B) DJI Matrice 100 UAV; (C) MAPIR V2 reflectance calibration ground target; (D) Crop Circle ACS $-430^{\circledR}$ sensor.

The collection of ground data was carried out using the Crop Circle ACS-430 ${ }^{\circledR}$ canopy sensor (Holland Scientific, Lincoln, USA) (Figure 1D). Unlike the Sequoia camera, Crop Circle ${ }^{\circledR}$ is an active sensor, as it emits its light source from a light-emitting diode (LED), with an emission frequency of $40,000 \mathrm{~Hz}$ and a field of view of $30^{\circ} \times 14^{\circ}$. This sensor captures the reflectance from the targets using three photodetectors in the red $(660-680 \mathrm{~nm})$, RE (720-740 nm), and NIR (770-790 nm) bands (Mulla, 2013). Despite not having bands with wavelengths coinciding with those of Sequoia, Crop Circle was used because it has similar band centers.

The flight plan was elaborated in the program Pix4D Capture, which was also used for the RPAS navigation, with longitudinal and lateral overlaps of $85 \%$ and $60 \%$ of the photographs, respectively, with a flight height of $100 \mathrm{~m}$ to obtain images with a ground sample distance (GSD) of $0.094 \mathrm{~m}$. The first flight was performed over Area A at 14:50 UTC (11:50 am local time), while the flight over Area B occurred one hour later (Figure 2). The duration of the flights took approximately $5 \mathrm{~min}$ and they were executed with no cloud cover in the surveyed areas.

The images from the Sequoia camera were taken before the collection of ground data at both studied areas because changes in the plant coverage due to walking and trampling may occur during the collection of reflectance data by the Crop Circle sensor.

The survey points of ground data by the Crop Circle ACS-430 sensor were defined from a $10 \times 10-\mathrm{m}$ sample grid created in a geographic information system (GIS) program (ArcMap v.10.3). The reflectance was measured on each point at a height of approximately $80 \mathrm{~cm}$ above the canopy, and the geographic coordinates of these points were measured by a Topcon double-frequency receiver (rover) by a quick-static Stop and Go survey (2 min at each point). High planimetric precision of these survey points and of photogrammetric control targets (i.e., ground control points) was achieved by correcting their coordinates in the Topcon Tools program by the relative post-processed method, using data from the GNSS base installed at EEA/UFRGS (latitude $-30.118775^{\circ}$ and longitude $-51.678630^{\circ}$; altitude $74.719 \mathrm{~m}$ ) and a GNSS station of the Brazilian Network for Continuous Monitoring (RBMC) of the Brazilian Institute of Geography and Statistics (IBGE), located in a building of the Department of Geodesy of the Federal University of Rio Grande do Sul (POAL Station, latitude $-30.074043^{\circ}$ and longitude $-51.119770^{\circ}$; altitude: $\left.77.533 \mathrm{~m}\right)$. The altimetric coordinates of the survey points and photogrammetric control targets were corrected by the IBGE MAPGEO2015 geoid undulation model. 


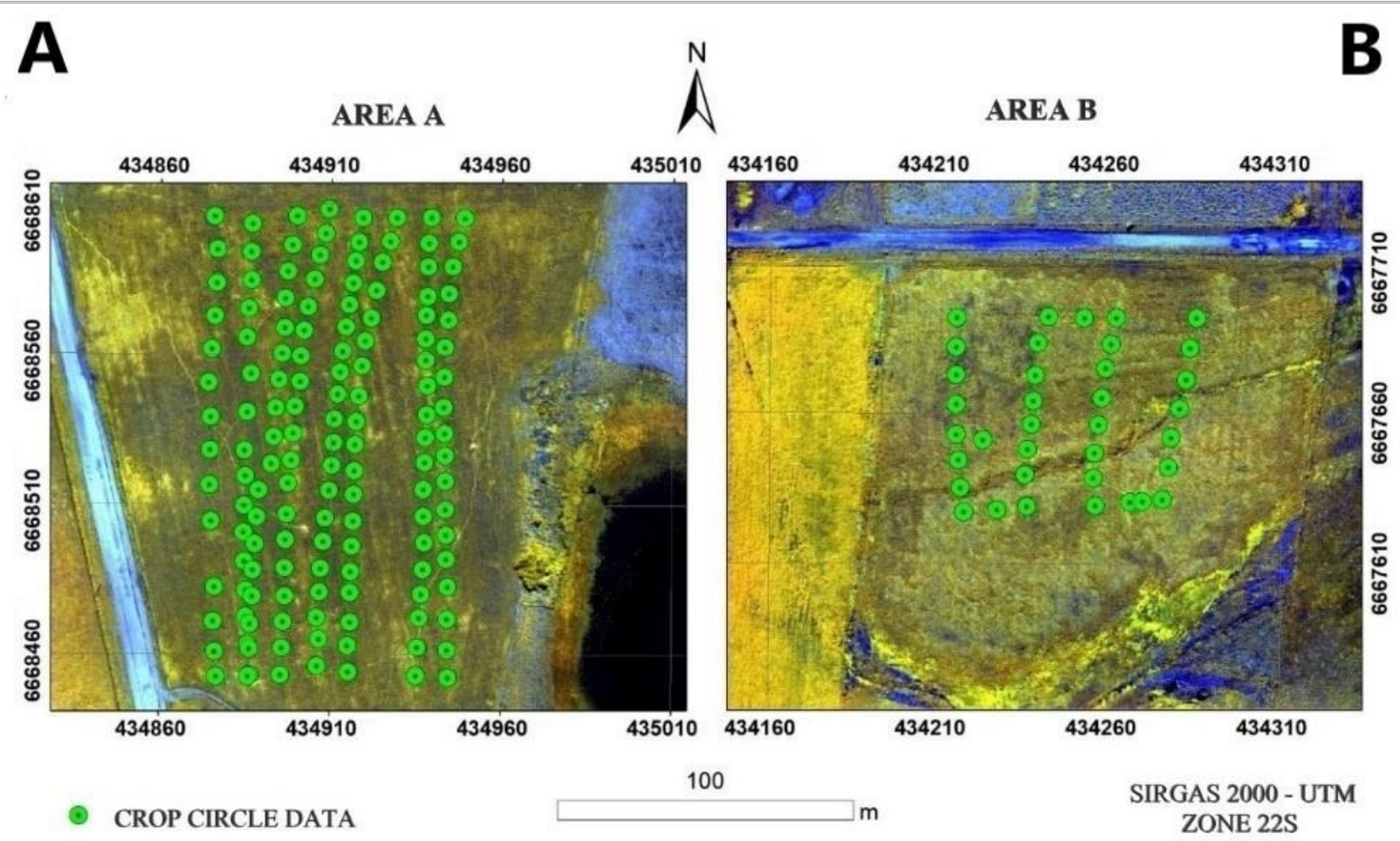

FIGURE 2. Distribution of the Crop Circle data over an orthophoto mosaic of Sequoia camera (RGB 432 color composite) in Areas A (2A) and B (2B).

The geometric processing of aerial images was carried out after the field survey using the software Agisoft Metashape 1.6, in which a photogrammetric project was created and the photographs were imported with Geotags containing the focal length of the camera, geographic coordinates, and ellipsoidal height referenced to the WGS84. The images were georeferenced using these metadata and the coordinates of eight photogrammetric control targets installed in Area A and five in Area B. The aerotriangulation in this software is performed using a scale-invariant feature transform (SIFT) algorithm, based on the search for similar features in the photographs, with an automatic tie point generation by image matching, and the application of structure from motion (SFM) and multiview stereo (MVS) techniques, resulting in a dense point cloud (Vera et al., 2017). The planialtimetric coordinates of the photo-identifiable control targets were used to adjust the georeferencing of the point cloud and photographs. The data of the planialtimetric point cloud are interpolated in this software by the triangulated irregular network (TIN) method to generate a digital elevation model (DEM). This 3D model was used for the orthorectification of the photographs and their resampling method was that of the nearest neighbor, used to preserve the values of digital numbers (16-bit images) so that the data could be used properly in the subsequent radiometric analyses. Finally, the geometrically rectified multispectral imagery was used to generate an orthophoto mosaic for Areas A and B. The software Metashape was also used for radiometric corrections of the orthophoto mosaic, first with the use of solar irradiance data from the sunshine sensor (SS) and then with pre-flight images taken from the MAPIR V2 calibration ground target (RC). The photographs taken with the shortest exposure time of the Sequoia camera (shutter 1/2,933.32) were used in this radiometric calibration to avoid brightness saturation by the lighter gray hues of the $\mathrm{RC}$.

Figure 2A shows the distribution of the 143 survey points collected using the Crop Circle sensor over the Sequoia orthophoto mosaics in Area A, while Figure 2B shows the 36 points collected in Area B. The planimetric root-mean-square error (RMSE) of the Crop Circle points in Areas A and B was $0.14 \mathrm{~m}$, while the altimetric RMSE was $0.15 \mathrm{~m}$ and $0.12 \mathrm{~m}$, respectively. The ground control points in the aerotriangulation of photographs presented a planimetric RMSE of $0.16 \mathrm{~m}$ (Area A) and $0.38 \mathrm{~m}$ (Area B) and an altimetric RMSE of $0.45 \mathrm{~m}$ for the orthophoto mosaic of both areas.

After importing the orthophoto mosaics into the Metashape, each of the spectral bands of interest (red, RE, and NIR) was selected, one of the RC targets (white, light gray, dark gray, or black) was identified, and the coverage area of each target was delimited separately in the MAPIR $\mathrm{RC}$ image. Then, the reflectance data were imported from the respective delimited target, which was used for the radiometric calibration of the image of each spectral band of the Sequoia camera. Two radiometric corrections were performed for each of the targets, one of them using the diffuse reflectance configuration parameters and the other using the total reflectance. Finally, radiometric corrections were carried out with the information from the sunshine sensor (SS), together with the data from the MAPIR RC.

The integer pixel values of the Sequoia camera images were converted into reflectance values (between 0 and 1) following the indications of Micasense (2019). This conversion was applied even to orthophoto mosaics without radiometric calibration to allow obtaining values comparable to those ones by the Crop Circle sensor to 
calculate the absolute and relative mean reflectance errors and spectral indices derived from these images.

The validation of reflectance values in the red, RE and NIR bands, and NDVI and NDRE spectral indices derived from orthophoto mosaics, generated by the different parameters used in the radiometric correction step, was performed using the values of these radiometric variables at the points measured with the Crop Circle sensor. The discrete data acquired by the Crop Circle was imported into the program ArcMap 10.3 as point shapefile. All orthophoto mosaics were imported into this program and, subsequently, the tool "extract multi-value by points" was used to extract the pixel values from these images into the point file database. Filtering of the Crop Circle data that presented more than one standard deviation of the point values of each experimental area was carried out before the comparison between the results of both sensors to eliminate incoherent reflectance values or spectral indices, following the methodology proposed by Menegatti \& Molin (2004) for yield data.

The mean absolute error (MAE), which is given by the sum of the difference in module between the estimated values and the observed values divided by the number of samples, was used as one of the statistical parameters to analyze the radiometric accuracy of the data. In this case, the observed values were the data obtained with the Crop Circle sensor and the estimated values were those of the orthophoto mosaics corrected by the different radiometric calibration parameters. The relative percentage errors were obtained by calculating the mean relative error (MRE), which is calculated by the ratio between the MAE and the observed value and multiplied by 100 to express the result in a percentage. Another measure adopted to evaluate the accuracy of orthophoto mosaics was the mean squared error (MSE), which is a measure that represents the standard deviation of the differences between the estimated and observed values. Other statistical parameters were adopted to describe the differences between the observed and estimated values: coefficient of residual mass (CRM), correlation coefficient ( $\mathrm{r}$ ), and Willmott index of agreement.

The CRM consists of the difference between the sum of the observed values and the sum of the estimated values, divided by the sum of the observed values. It can present values that vary between $-\infty$ and $+\infty$, with the optimal result being zero (indicating an ideal adjustment); positive values indicate the underestimation of the predicted values, while negative values indicate their overestimation (Bonfante et al., 2010). The correlation coefficient ( $r$ ) estimates the degree of linear correlation between two quantitative variables, in which the result is a dimensionless index with values between -1 and 1 , with values close to zero indicating the absence of correlation (Addiscott \& Whitmore, 1987). The index of agreement developed by Willmott is designated by the letter " $\mathrm{d}$ " and represents the precision and the degree of discrepancy between the actual data (in this case, those obtained by the Crop Circle sensor) and the estimated values (measured from Sequoia camera). It can vary from 0 to +1 , and values close to or equal to +1 indicate perfect agreement between the data, while values close to zero indicate total disagreement (Willmott et al., 2012). The coefficient "c" was used as a confidence or performance indicator of the estimated data, calculated from the product between the index of agreement and the correlation coefficient ( $c=d x$ r). Values of "c" lower than 0.40 represent low reliability and values higher than 0.85 indicate excellent confidence in the estimated data (Camargo \& Sentelhas, 1997).

\section{RESULTS AND DISCUSSION}

The statistical results of MAE, standard deviation, and MRE for the comparison between spectral reflectance and VIs obtained by Sequoia and Crop Circle for Areas A and $\mathrm{B}$ are shown in Figure 3. 

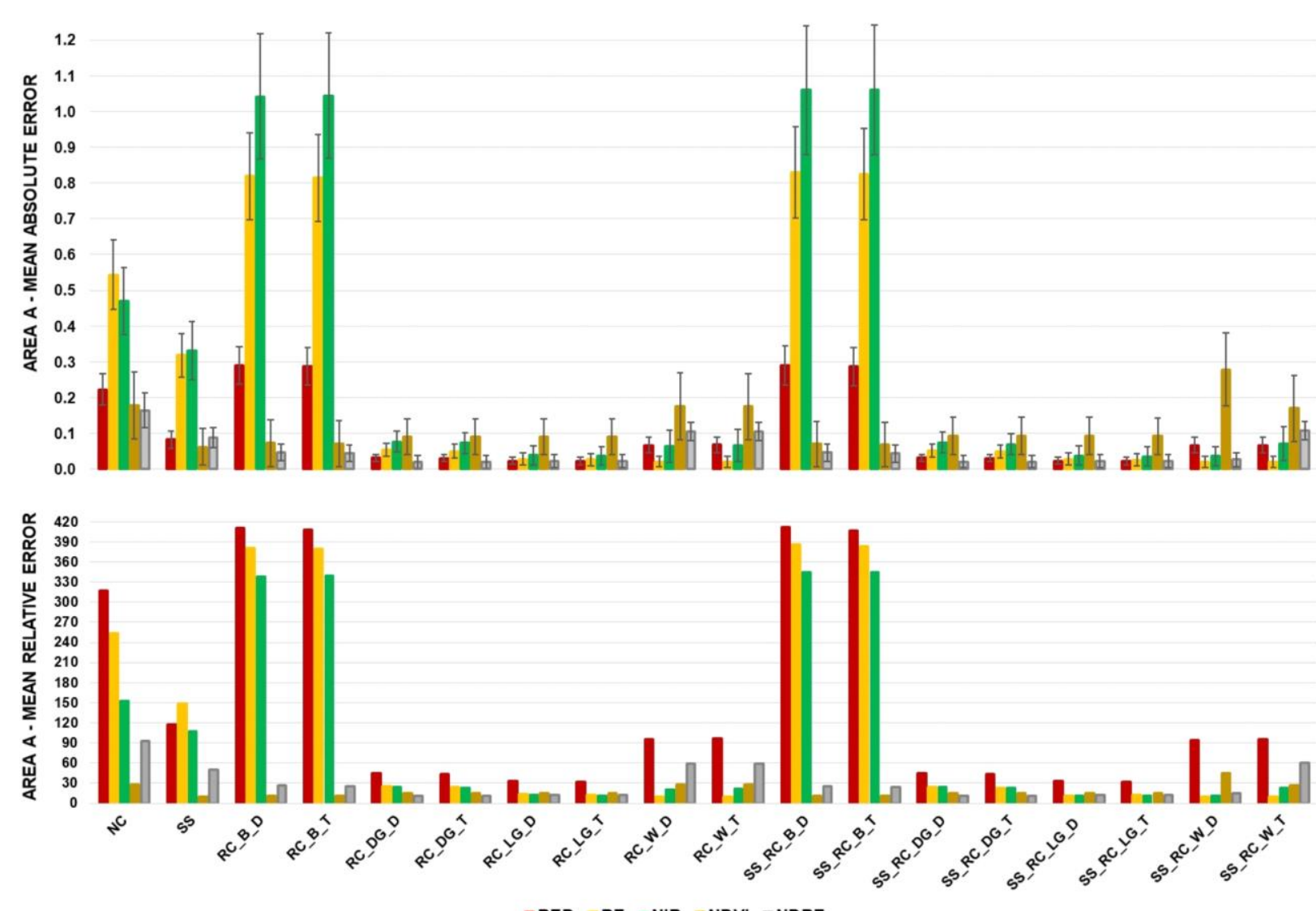

$\because$ RED $\approx$ RE $=$ NIR $=$ NDVI $\square$ NDRE
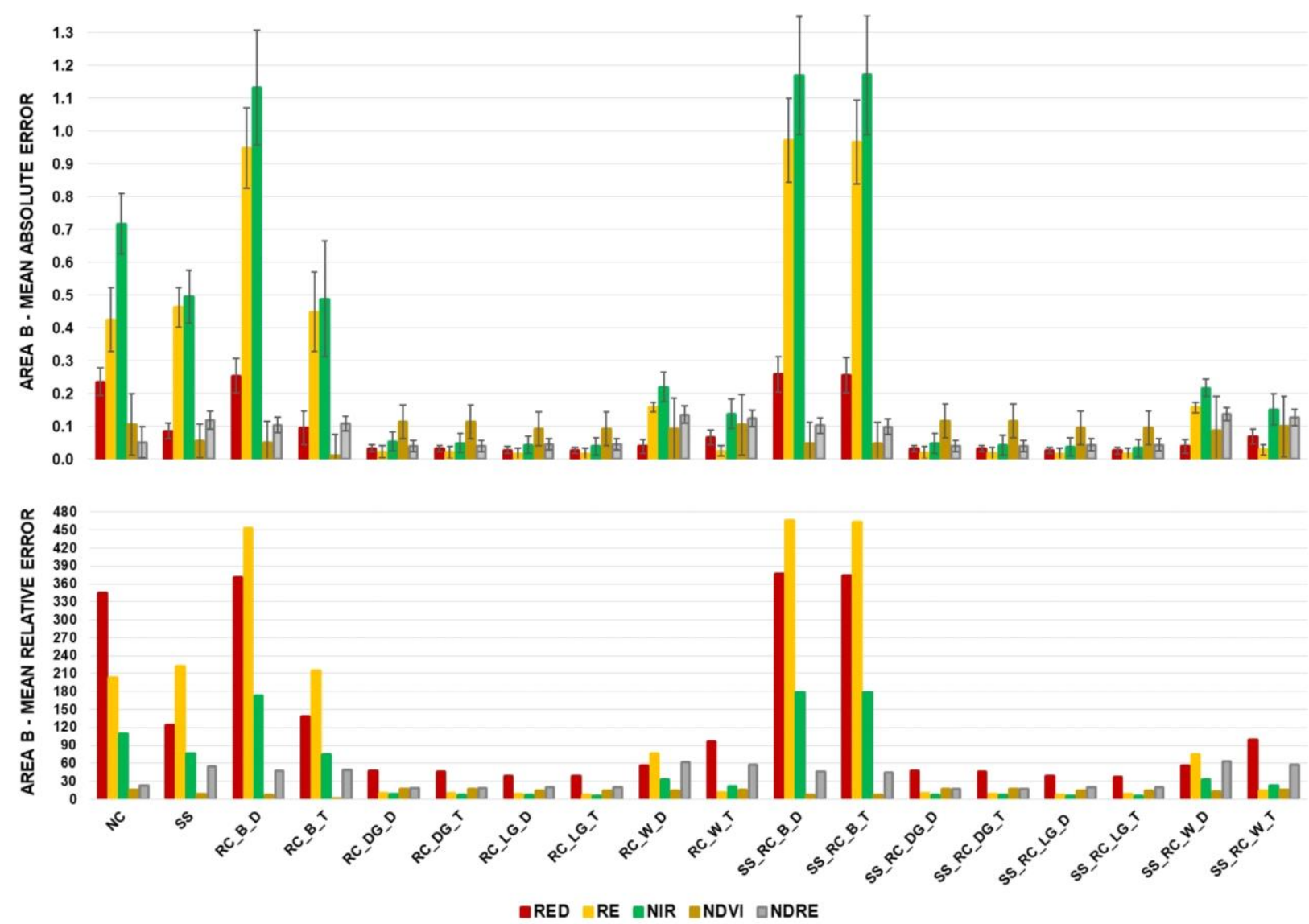

FIGURE 3. Mean absolute error and standard deviation, and mean relative error of orthophoto mosaics from Areas A and B, processed with no radiometric calibration (NC), using the Sunshine Sensor (SS) data and/or data from different references of the MAPIR reflectance calibration ground target (RC: $\mathrm{B}=$ black; $\mathrm{DG}=$ dark gray; $\mathrm{LG}=$ light gray; $\mathrm{W}=$ white; $\mathrm{D}=$ diffuse; $\mathrm{T}=$ total). 
Inaccurate NDVI data result, for example, in errors in estimates of vegetation density, its development and vigor, of plant biomass, phytosanitary status and productive potential of the crop, and delimitation of management zones in precision agriculture (Damian et al., 2020), in addition to inaccurate estimates for input application at variable rates, such as pesticides, irrigation, and topdressing nitrogen fertilization (Castro \& Inamasu, 2014; Turra, 2016).

The NDRE is recommended for areas where there is interference from soil reflectance at low canopy densities. Moreover, it is sensitive to changes in the chlorophyll content of leaves in canopies at advanced stages of development, with leaf area index (LAI) values higher than 2 (Mulla, 2013). However, the low accuracy of NDRE measurements using these calibration procedures leads to problems in the traditional application of this index, such as the identification of vegetation types, chlorophyll content, and nutritional and phytosanitary status (Doumit \& Kiselevm 2017).

Figure 3 shows that the images corrected with RC showed significant changes only with a variation in the targets (light gray, dark gray, and black), with no changes in their diffuse or total reflectance settings, as these changes were only detected from $10^{-3}$, except for the white target, which had small variations in reflectance as the target setting (diffuse and total) changed. The results obtained with only RC or RC plus SS were very similar, with no significant changes in the results. Therefore, the results are considered similar in this case and hence the discussion considers only RC targets.

The black target showed unsatisfactory results for the calibration of the orthophoto mosaics of spectral bands for both study areas. The red band presented a MAE value equal to 0.3 , whereas the RE and NIR bands reached values of $0.095 \pm 0.09$ and $1.04 \pm 0.18$, respectively. On the other hand, the results obtained in VIs were closer to the data measured with the Crop Circle, i.e., the orthophoto mosaic of NDVI and NDRE had MAE values equal to $0.07 \pm 0.07$ and $0.05 \pm 0.02$, respectively.

The orthophoto mosaic errors of spectral bands corrected from the black target may be related to the low brightness characteristics of the RC image, which was taken by the Sequoia camera in short exposure time, thus impairing the detection of the black target. On the contrary, the good correlation achieved with VIs is due to the proportion of similar errors of the spectral bands, which is compensated by the arithmetic of bands used for their calculation.

The results of orthophoto mosaics obtained using the dark gray target showed gains in radiometric accuracy for both the spectral bands and the evaluated VIs, in which the MRE values were lower than 50\% in the spectral bands for both areas and, in some cases, reaching optimal results, such as in Area B, with MRE values of $48 \%$ (red band), $10 \%$ (RE), $8 \%$ (NIR), 17\% (NDVI), and 18\% (NDRE).

The best results were obtained for the multispectral orthophoto mosaic corrected from the light gray target. The orthophoto mosaic of the red spectral band in Area A had an MAE value of $0.02 \pm 0.01$, an MSE close to zero, and an MRE of $33 \%$. Similar results of MAE and MSE were also observed for Area B, which showed a higher MRE, with a value of only $38 \%$. The orthophoto mosaic of Area $\mathrm{A}$ had low MAE in the RE $(\mathrm{MAE}=0.03 \pm 0.02$;
$\mathrm{MRE}=13 \%)$ and NIR bands $(\mathrm{MAE}=0.04 \pm 0.03 ; \mathrm{MRE}=$ $13 \%)$. The same behavior was observed for the orthophoto mosaic of Area B (RE with MAE $=0.02 \pm 0.01$ and MRE $=8 \%$; NIR with MAE $=0.04 \pm 0.03$ and $\mathrm{MRE}=7 \%$ ). Likewise, only VIs showed similar results to those measured in the field. The NDVI orthophoto mosaics of Areas A and B had low MAE (0.09 \pm 0.05$)$ and MSE (0.01), with an MRE value of $14 \%$. Overall, the NDRE for this target presented the best results relative to the field data. The orthophoto mosaic of Area A, for example, had an MAE value equal to $0.02 \pm 0.02$, MSE close to zero, and MRE of $12 \%$. Also, Area B showed similar errors, but with an MRE value slightly higher $(21 \%)$, which is still considered low (Figure 3).

In general, the white reference target presented intermediate MAE and MRE results, i.e., below the results achieved with the light gray target. However, this target showed a significant difference when changing the diffuse or total reflectance settings.

The mean absolute and relative errors in the red spectral band were probably due to the difference in its width in both sensors because, unlike the Crop Circle sensor, the Sequoia camera can register chlorophyll B absorption processes at $642 \mathrm{~nm}$. The spectral response of the vegetation is directly related to the surface properties and cellular structure of leaves (mesophyll), being also conditioned to concentrations and distributions of biochemical components, such as photosynthetic pigments (Ogaya \& Peñuelas, 2003). Thus, EMR interacts differently in each of the spectral bands. For instance, the absorption processes of EMR at visible wavelengths by leaf pigments that act in the photosynthesis and photoconversion processes. These absorptions depend on plant characteristics and have absorption peaks at different bands in the electromagnetic spectrum. For instance, the absorption caused by chlorophyll $\mathrm{A}$ in the red spectral band occurs at wavelengths of 630 and $662 \mathrm{~nm}$; chlorophyll B has its absorption peaks at $642 \mathrm{~nm}$ (Gross, 1991; Mulla, 2013).

The RE spectral band shows an increase in the vegetation reflectance relative to the visible. The increase in reflectance is significant in the NIR spectral band due to the EMR scattering processes in the palisade mesophyll, where photosynthetic chloroplasts and pigments are located, and spongy mesophyll, characterized by intercellular spaces with gases and water (Gross, 1991). The mean absolute and relative errors in RE and NIR were also the results of the difference between the width of these bands in the Crop Circle sensor and Sequoia camera. Thus, these sensors have different sensitivity to photosynthetic pigments in the RE band and to mesophyll characteristics in the NIR band. Other important factors that contributed to errors in the orthophotos of the analyzed bands were the difference in FOV and height of acquisition of these sensors, as the suborbital covers a larger area in each field of view and is more sensitive to LAI, which has an influence on the red radiation absorption process by plants. These errors were relatively lower in the NIR band, in which LAI and the EMR scattering processes in the mesophyll should be considered.

The location and magnitude of absolute errors (AEs) of NDVI and NDRE of the orthophoto mosaic obtained from the radiometric correction with the light gray target are represented in the thematic maps of Figure 4. 

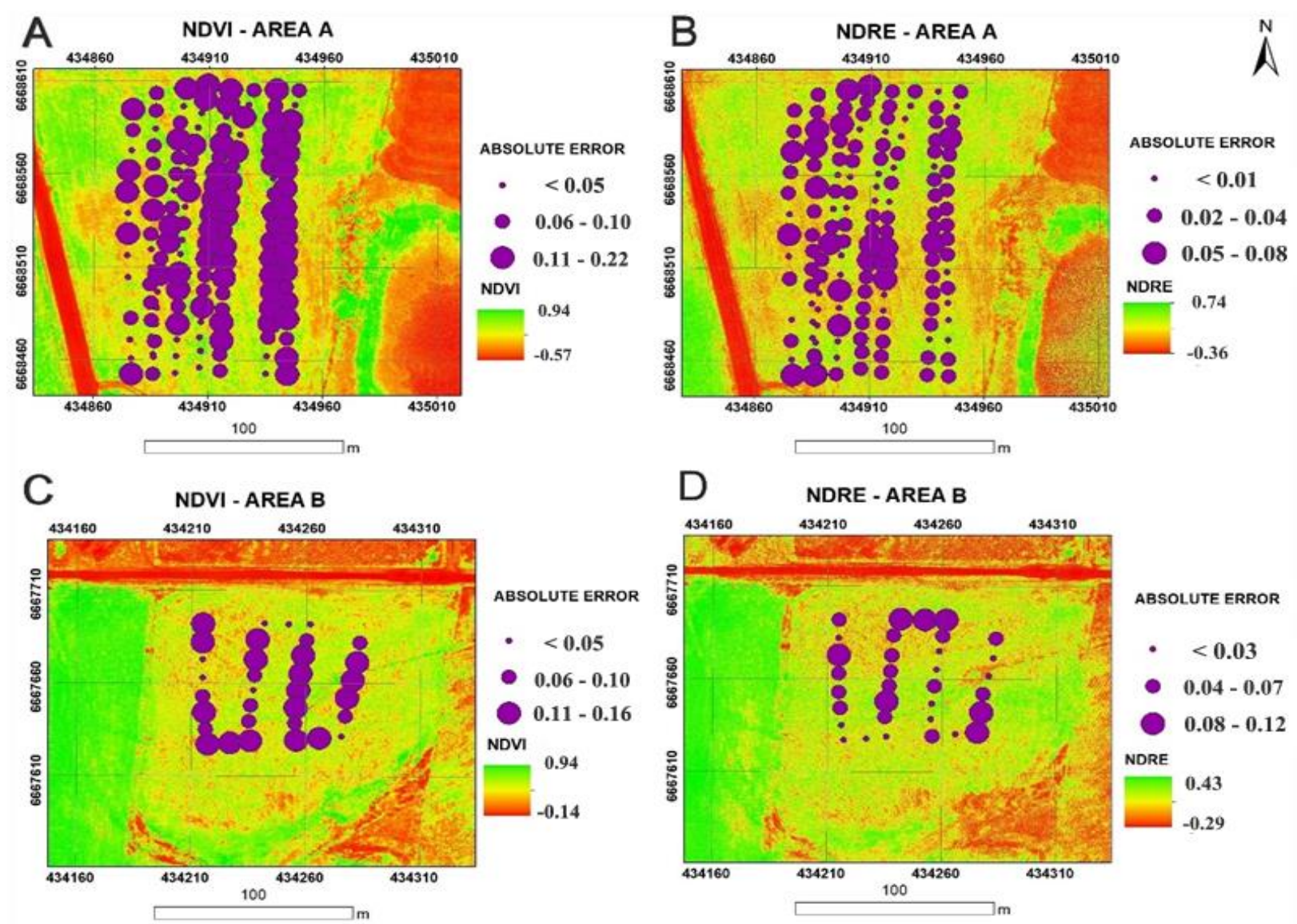

FIGURE 4. NDVI and NDRE absolute errors of the orthophoto mosaics processed with the light gray reference of the MAPIR reflectance calibration ground target. The ground truth data used for this error analysis were obtained from the Crop Circle sensor. (A) NDVI absolute errors in Area A; (B) NDRE absolute errors in Area A; (C) NDVI absolute errors in Area B; (D) NDRE absolute errors in Area B.

The NDVI orthophoto mosaic from Area A (Figure $4 \mathrm{~A})$ showed maximum variation of absolute error (AE) from 0.11 to 0.22 , which represented $68 \%$ of the thematic classes, with its highest concentration in the east. These AEs showed an intermediate linear correlation $(r=0.46)$ with the NDVI values of this image. Moreover, the NDRE orthophoto mosaic (Figure 4B) showed a range of maximum $\mathrm{AE}$ values from 0.05 to 0.08 (14\% of the data), with a negative linear correlation $(\mathrm{r}=-0.54)$ with the NDRE values. The most frequent AEs in the NDVI orthophoto mosaic of Area B (Figure 4C) varied from 0.11 to 0.16 , representing $52 \%$ of the errors. A high linear correlation $(r=0.86)$ with AEs was observed for this data set, indicating that the errors increased in areas with higher NDVI values and, consequently, higher plant biomass and LAI. In addition, the NDRE orthophoto mosaic of Area B (Figure 4D) showed a high negative linear correlation ( $\mathrm{r}=$ $-0.85)$ with these errors.

Figure 5 describes the distribution and relationships between the NDVI and NDRE values obtained from direct measurements made by the Crop Circle sensor and the values of these indices derived from the orthophoto mosaic with higher radiometric accuracy, calibrated by the light gray target of the MAPIR V2 reflectance calibration ground target. 

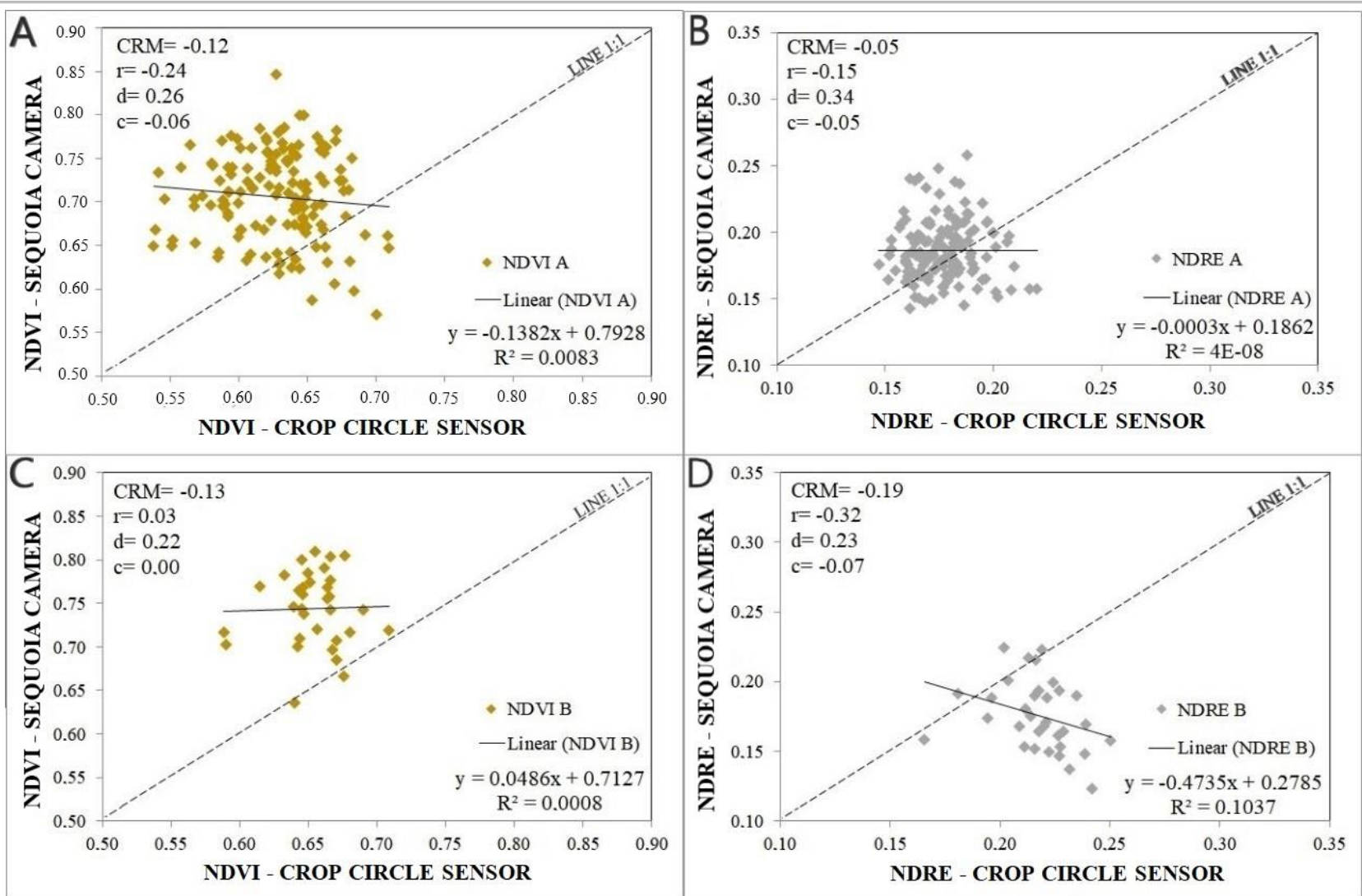

FIGURE 5. Relationships between NDVI and NDRE data measured from the Crop Circle sensor and derived from the orthophoto mosaics processed with the light gray reference of the MAPIR reflectance calibration ground target: (A) NDVI data in Area A; (B) NDRE data in area A; (C) NDVI data in Area B; (D) NDRE data in Area B.

Areas $\mathrm{A}$ and $\mathrm{B}$ showed negative CRM values $(-0.12$ and -0.13 , respectively), which indicate a slight overerestimation of the NDVI values in the orthophoto mosaic. The correlation coefficient of the NDVI of Area A was also negative, with values close to zero $(r=-0.24)$. On the other hand, the correlation coefficient of the NDVI of the orthophoto mosaic was positive, with values close to zero $(\mathrm{r}=0.03)$. It indicates a low correlation between the variables in both areas. The values of the index of agreement " $d$ " were close to zero $(\mathrm{d}=0.26)$ in Area A, with similar results $(d=0.22)$ for area $B$. The confidence indicator " $\mathrm{c}$ " for the NDVI in both areas presented low values, that is, 0.06 in Area A and 0.00 in Area B, indicating the low performance of the linear model. These low values of linear agreement may be explained by differences in the instantaneous field of view (IFOV) and operating principle of the sensors, one of them being active (Crop Circle) and the other passive (Sequoia camera).

Regarding the NDRE, Area A showed a low CRM $(-0.05)$ value, which was higher for the data in Area B $(-0.19)$, with these negative values indicating an overestimation of this index in the orthophoto mosaic. The correlation coefficient values for the NDRE of both areas were negative and close to zero (Area $\mathrm{A}=-0.15$ and Area $\mathrm{B}=-0.32$ ). The index of agreement " $d$ " indicated a high discrepancy between the values obtained by the Crop Circle sensor compared to the Sequoia camera data for both study areas (Area $\mathrm{A}=0.34$ and Area $\mathrm{B}=0.23$ ). The correlation coefficient indicated the low performance of the linear model (Area A with $r=-0.05$ and Area B with $\mathrm{r}=-0.07)$

\section{CONCLUSIONS}

The orthophoto mosaics generated with no radiometric calibration procedures showed significant errors for the red, RE and NIR spectral bands, and NDVI and NDRE indices. The exclusive use of the solar irradiance sensor (sunshine sensor) data of the Sequoia camera is not recommended in the radiometric calibration of images to measure the reflectance in these spectral bands and to calculate the NDVI and NDRE indices, although it has improved the radiometric accuracy of the orthophoto mosaic. A low linear correlation was observed between the values of these spectral indices measured with the Crop Circle sensor and values derived from orthophoto mosaics.

Using a reflectance calibration ground target (RC) is essential in studies that require high radiometric accuracy of the data. The data of diffuse and total reflectance from MAPIR V2 RC produced images with radiometric accuracy very similar to the data from the Crop Circle sensor, with differences in reflectance in the order of only $10^{-3}$. The best result with the exposure time $1 / 2,933.32 \mathrm{~s}$ was obtained with the light gray target. It may have occurred due to the characteristics of the low brightness and contrast of the image taken by the camera for radiometric calibration, which ended up saturating the darker RC targets. The use of the light gray target in the radiometric calibration resulted in orthophotos with very low mean squared error (lower than 0.01) for all analyzed bands and spectral indices. 


\section{ACKNOWLEDGMENTS}

The authors would like to thank the Coordination for the Improvement of Higher Education Personnel (CAPES) for the scholarship granted to the first author, and the Eng. Mirayr Raul Quadros de Souza for the RPAS operation.

\section{REFERENCES}

Addiscott TM, Whitmore AP (1987) Computer simulation of changes in soil mineral nitrogen and crop nitrogen during autumn, winter and spring. The Journal of Agricultural Science 109(1):141-157. DOI: http://dx.doi.org/10.1017/S0021859600081089

Barnes EM, Clarke TR, Richards SE, Colaizzi PD, Haberland J, Kostrzewski M, Waller P, Choi C, Riley E, Thompson T, Lascano RJ, Li H, Moran MS (2000) Coincident detection of crop water stress, nitrogen status and canopy density using ground-based multispectral data. International Conference on Precision Agriculture. Madison, American Society of Agronomy, Proceedings..

Bonfante A, Basile A, Acutis M, De Mascellis R, Manna P, Perego A,Terribile F (2010) SWAP, CropSyst and MACRO comparison in two contrasting soils cropped with maize in Northern Italy. Agricultural Water Management 97(7):1051-1062. DOI:

http://dx.doi.org/10.1016/j.agwat.2010.02.010

Camargo AP, Sentelhas PC (1997) Performance evaluation of different potential evapotranspiration estimating methods in the state of São Paulo, Brazil. Revista Brasileira de Agrometeorologia 5(1):97-98.

Castro JLA, Inamasu RY (2014) Uso de veículos aéreos não tripulados (VANT) em agricultura de precisão. In: Agricultura de precisão: resultados de um novo olhar. Livro Técnico-Científico, Embrapa Instrumentação, 109-134.

Damian JM, Pias OHD, Cherubin MR, Fonseca AZ, Fornari EZ, Santi AL (2020) Applying the NDVI from satellite images in delimiting management zones for annual crops. Scientia Agricola 77(1):1-11. DOI: http://dx.doi.org/10.1590/1678-992x-2018-0055

Deng L, Mao Z, Li X, Hu Z, Duan F, Yan Y (2018) UAVbased multispectral remote sensing for precision agriculture: A comparison between diferent cameras. ISPRS Journal of Photogrammetry and Remote Sensing (146):124-136. DOI:

https://doi.org/1016/j.isprsjprs.2018.09.008

Doumit J, Kiselevm E (2017) Comparison of divert vegetation indices for agricultural mapping over the Krasnodar region. Internacional Congress Geo-Tunis 5(15):11-20.

Gross J (1991) Pigments in vegetables chlorophylls and carotenoids. New York, Van Nostrand Reinhold.
MAPIR (2020) MAPIR Camera Reflectance Calibration

Ground Target Package (V2) Available:

https://www.mapir.camera/collections/survey-cameraaccessories/products/ mapir-camera-reflectance-calibrationground-target-package-v2. Accessed: Jun 10, 2020.

Menegatti L, Molin JP (2004) Remoção de erros em mapas de produtividade via filtragem de dados brutos. Revista Brasileira de Engenharia Agrícola e Ambiental 8(1): 126-134. DOI: https://doi.org/10.1590/S1415-43662004000100019

Micasense (2019) In: What are the units of the Atlas GeoTIFF output? Avaliable:

https://support.micasense.com/hc/enus/articles/215460518-What-are-the-units-of-the-AtlasGeo TIFF-output-. Accessed: Aug 20, 2020.

Mulla D (2013) Twent five years of remote sensing in precision agriculture: Key advances and remaining knowledge gaps. Biosystems Engineering (114):358-371. DOI: http://dx.doi.org/10.1016/j.biosystemseng.2012.08. 009

Ogaya, R, Peñuelas J (2003) Comparative field study of Quercus ilex and Phillyrea latifolia: Photosynthetic response to experimental drought conditions. Environmental and Experimental Botany 2(50):137-148. DOI: https://doi.org/10.1016/S0098-8472(03)00019-4

Pereira S, Silva O, Denser V, Pamboukian SVD (2016) Sensoriamento remoto aplicado à agricultura de precisão no cultivo de bambu. Revista Mackenzie de Engenharia e Computação 16(1): 8-33.

Rouse JW, Haas RH, Schell JA, Deering DW (1974) Monitoring vegetation systems in the great plains with ERTS. In: Earth Resources Technology Satellite (ERTS) symposium. Greenbelt, NASA.

Puri V, Nayyar A, Raja L (2017) Agriculture drones: A modern breakthrough in precision agriculture (20):507518. DOI:

https://doi.org/10.1080/09720510.2017.1395171

Turra MA (2016) Manejo do nitrogênio em cultivares de trigo baseado no índice de vegetação por diferença normalizada (NDVI) Dissertação, Porto Alegre, Universidade Federal Do Rio Grande Do Sul.

Vera CD, Munizaga MA, Icaza MJ, Herrera NM, Méndez AR (2017) A photogrammetry software as tool for precision agriculture: A case Study 282-295. DOI: https://doi.org/10.1007/978-3-319-67283-0_21

Willmott CJ, Robeson SM, Matsuura K (2012) Short Communication: A refined index of model performance. International Journal of Climatology 32(13):2088-2094. DOI: https://doi.org/10.1002/joc.2419

Zadoks J, Chang T, Konzak C (1974) A decimal growth code for the growth stages of cereals. Weed Research 14(6):415-421. 